\title{
Mammalian Skeletogenesis and Extracellular Matrix: What can We Learn from Knockout Mice?
}

\author{
Attila Aszódi, ${ }^{1 *}$, John F. Bateman², Erika Gustafsson', Ray Boot-Handford, ${ }^{\text {,* }}$, and Reinhard Fässler1 \\ ${ }^{1}$ Department of Experimental Pathology, Lund University, 22185 Lund, Sweden and ${ }^{2}$ Department of \\ Paediatrics, University of Melbourne, Victoria 3052, Australia
}

\begin{abstract}
Formation of the vertebrate skeleton and the proper functions of bony and cartilaginous elements are determined by extracellular, cell surface and intracellular molecules. Genetic and biochemical analyses of human heritable skeletal disorders as well as the generation of knockout mice provide useful tools to identify the key players of mammalian skeletogenesis. This review summarises our recent work with transgenic animals carrying ablated genes for cartilage extracellular matrix proteins. Some of these mice exhibit a lethal phenotype associated with severe skeletal defects (type II collagen-null, perlecan-null), whereas others show mild (type IX collagen-null) or no skeletal abnormalities (matrilin-1-null, fibromodulin-null, tenascin-C-null). The appropriate human genetic disorders are discussed and contrasted with the knockout mice phenotypes.
\end{abstract}

Key words: skeletogenesis/extracellular matrix/skeletal disorders/knockout mice

Development of the mammalian skeleton is a temporally and spatially coordinated process, which is initiated by the condensation and differentiation of mesenchymal progenitor cells. Progenitors from the neural crest generate the craniofacial skeleton, sklerotomal cells form most elements of the axial skeleton, and the lateral plate mesoderm gives rise to the appendicular skeleton. Bone can develop via two basic mechanisms: mesenchymal precursor cells either differentiate directly into bone matrix-producing osteoblasts (intramembranous ossification) or first form a cartilaginous intermediate which is subsequently replaced by bone (endochondral ossification). The first mechanism is responsible for the formation of the flat bones of the skull, much of the facial skeleton, a part of the clavicle, and the cortical bone shaft of the long bones. The second mechanism occurs during the development of other bones such as long bones of

\footnotetext{
* To whom correspondence should be addressed: Department of Experimental Pathology, Lund University, 22185 Lund Sweaden.

Tel: +46-46-173-553,

E-mail: attila.aszodi@pat.lu.se

** On research leave from School of Biological Sceinces, University of Manchester, Manchester M13 9PT, UK.

Abbreviations: ECM, extracellular matrix; GAG, glycosaminoglycan; IVD, intervertebral disc; HS, heparan sulfate; COMP, cartilage oligomeric protein; ER, endoplasmatic reticulum; FGF, fibroblast growth factor, FGFR3, fibroblast growth factor receptor-3; CAPs, collagen fibril-associated proteins; vWFA, von Willebrand factor A; EGF, epidermal growth factor; LRPS, leucine-rich proteoglycans.
}

the legs, vertebrae and ribs.

Skeletal morphogenesis is characterized by the expression of a special set of extracellular matrix proteins and is tightly regulated via transcription factors, signaling molecules, hormones and local growth factors (Erlebacher et al., 1995). Our knowledge of how these molecules and factors act on skeletogenesis continuously increases, which not only helps to understand the normal development of skeletal elements but also contributes to a better understanding of skeletal diseases. During the last two decades human genetics has successfully identified gene mutations for many hereditary skeletal disorders resulting in osteochondrodysplasias or altered bone homeostasis (reviewed by Mundlos and Olsen, 1997a, 1997b). Furthermore, the conventional and conditional gene targeting experiments in mice have opened a new window which allow us to clarify the role of a particular gene product in skeletal development. The establishment of transgenic mouse strains with defined genetic alterations can also provide animal models for human skeletal disorders and might help to develop therapeutic tools for the treatment of various diseases affecting the skeleton.

In this review we first shortly summarise the basic morphogenetic steps of endochondral bone formation and secondly give an overview of knockout mice with minimal or complex skeletal phenotypes generated in our laboratory (see Table I). 

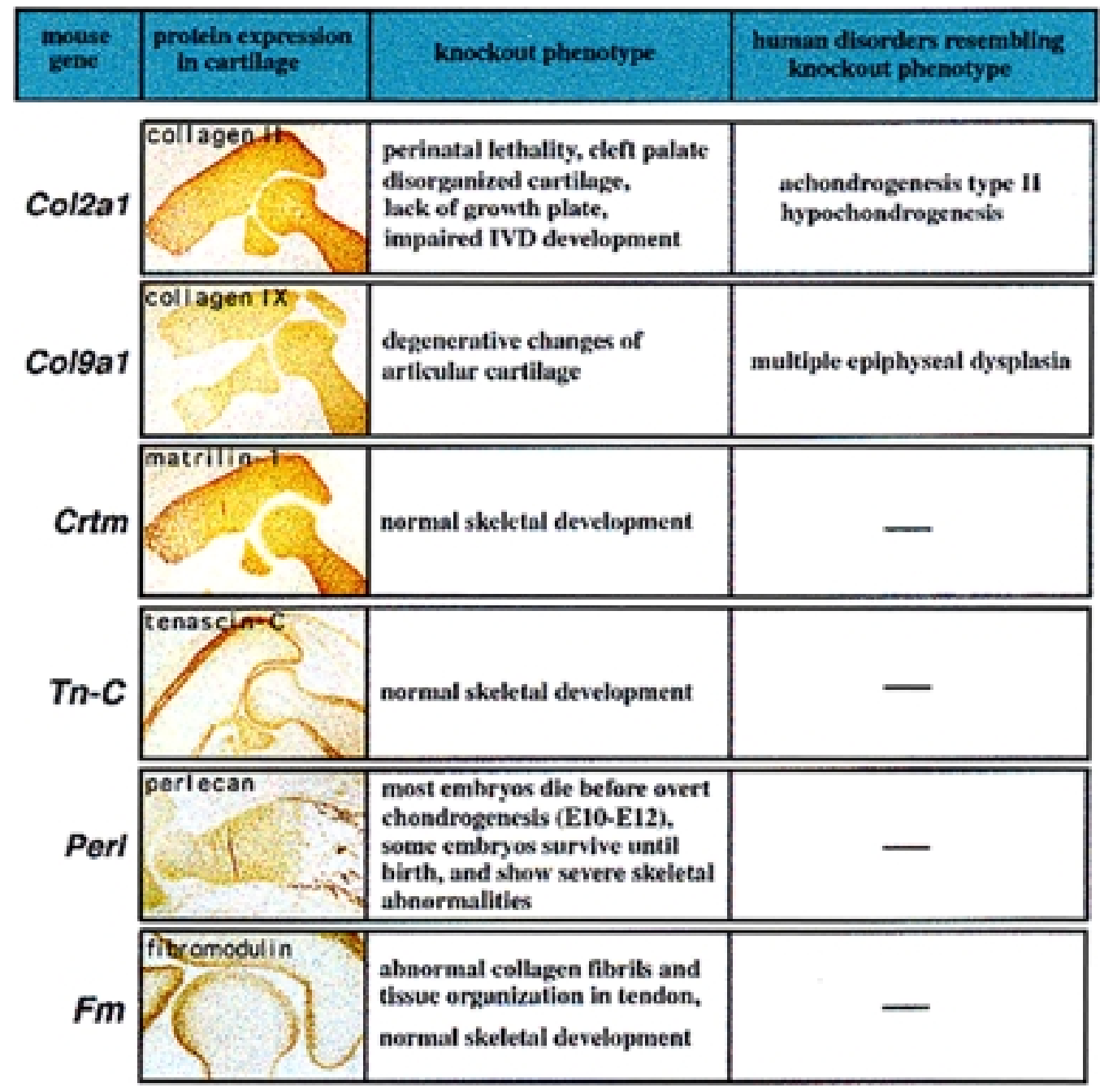

\section{Morphogenetic sequence of endochondral ossification}

The histological events of the endochondral bone formation (Fig. 1) are particularly well studied in the case of long bones (Horton, 1993). The initial cartilaginous anlage is completely avascular and contains rounded chondrocytes which secrete cartilage-specific matrix proteins and divide rapidly. At the periphery of the cartilage model, cells form the perichondrium and proliferate contributing to the lateral growth of the anlage. The intensive interstitial and appositional growth results in the enlargement of the anlage and its separation into a middle shaft (diaphysis) and club-shaped expansions at the ends (epiphyses). Later, in the central part of the diaphysis, the chondrocytes undergo a maturation process leading to the differentiation of hypertrophic chondrocytes. Simultaneously, at the mid-shaft, the osteoprogen- itor cells of the perichondrium differentiate into osteoblasts and lay down osteoid to form a bony collar around the diaphysis. Once the bone collar is formed, the matrix around the hypertrophic chondrocytes is mineralized and prepared for vascularisation. Capillaries grow through the periosteal shaft accompanied by undifferentiated mesenchymal and hemopoietic progenitor cells. Some of these cells differentiate into osteoblasts, others into chondroclasts, osteoclasts and the calcified cartilage is gradually resorbed and replaced by a network of trabecular bone. In addition to this primary ossification, after birth, blood vessels invade the epiphyseal ends of the bone forming secondary ossification centers. Closest to the epiphyseal surface the cartilage is not replaced by bone, remains intact and persists permanently as articular cartilage.

The longitudinal growth of the bone is attributed to a specialized cartilage structure, called growth plate, between the 

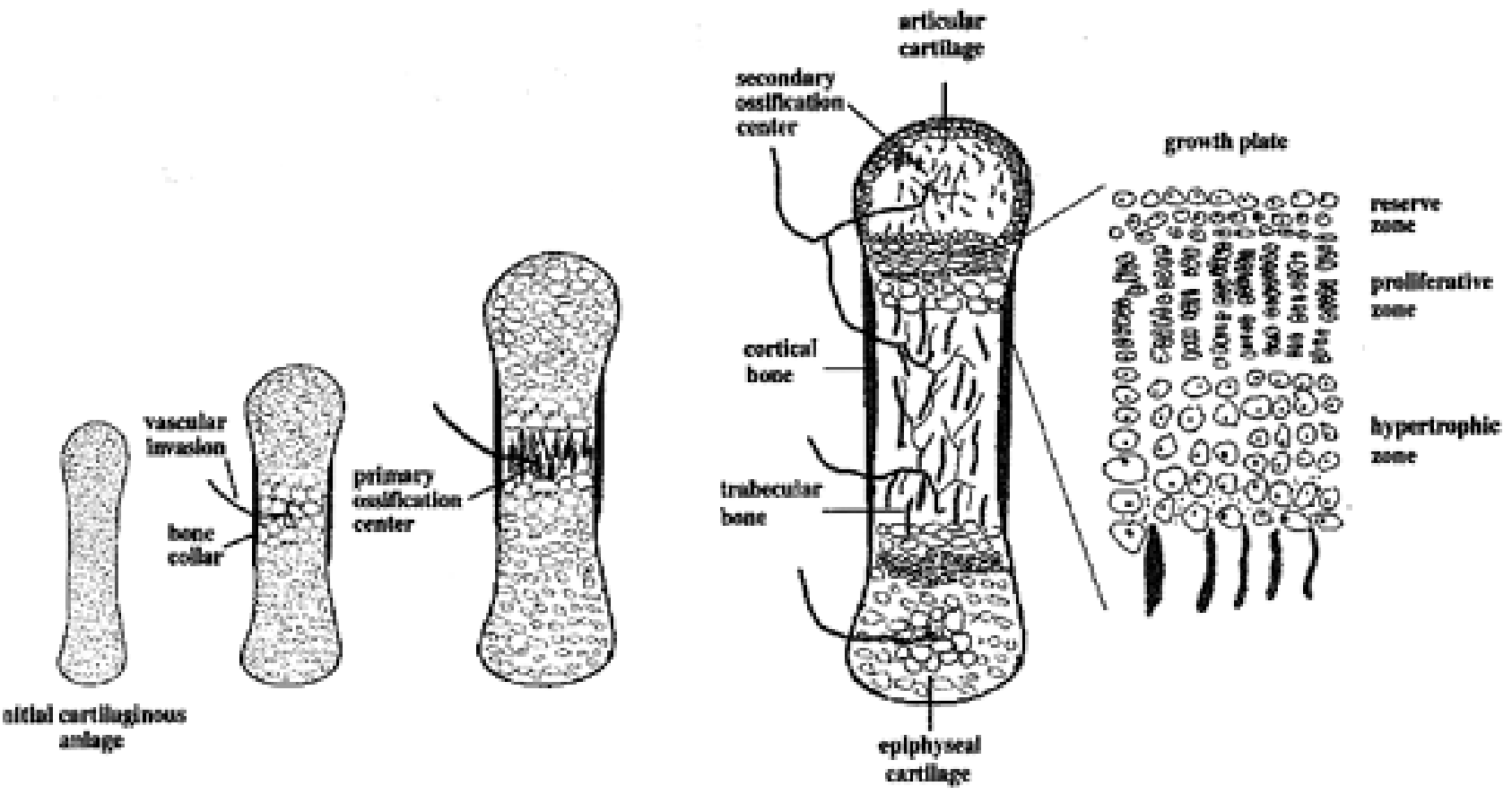

Fig. 1. Morphogenetic events of endochondral bone formation. During embryogenesis cartilaginous models are formed by the condensation of committed mesenchyme. The cartilage anlage is later mineralized and replaced by bone through endochondral ossification. The linear skeletal growth primary takes place at the growth plate where the chondrocytes are organized into discrete vertical zones. For details see text.

epiphysis and diaphysis. Growth plate chondrocytes form columns with discrete zones of morphologically and metabolically distinct cells (Hunziker, 1994). The zone farthest from the diaphysis is the region of resting or reserve chondrocytes. These small, isotrophic cells rarely divide and may constitute a stem cell pool for the cartilage columns. Those cells which enter the cell cycle undergo clonal expansion and generate the proliferative zone containing flattened chondrocytes. Kinetic studies on murine growth plate indicate that only cells in the upper half of the proliferative zone actually divide (Vanky et al., 1998), whereas cells in the lower part undergo a maturation process. Chondrocytes near to the epiphysis-diaphysis junction (metaphysis) increase in size and form the hypertrophic zone. The fate of hypertrophic chondrocytes is controversial: it is generally believed that terminal cells at the cartilage-bone border degenerate and die due to necrosis or programmed cell death (apoptosis). However, it has been shown by improved fixation and stereological methods that hypertrophic chondrocytes can be morphologically intact and metabolically active even adjacent to the invading blood vessels (Hunziker et al., 1984, 1987). Furthermore, there is increasing evidence that hypertrophic chondrocytes close to the perichondrial/periosteal osteogenic cells might differentiate into osteoblasts instead of undergoing apoptosis (for review see Bianco et al., 1998).

Whereas the longitudinal growth of bones is primarily achieved via proliferation, matrix production and hypertrophy of growth plate chondrocytes (Hunziker, 1994), the increase in diameter is regulated by different processes. At the diaphysis, the outer surface of the cortical bone (periosteum) deposits new bone, whereas the inner surface (endosteum) resorbs it. However, at the conically shaped metaphyses of certain long bones, the cortex is resorbed by the periosteum and new bone is deposited by the endosteum. The actual balance between deposition and resorption determines the thickness of cortical bone (Schenk et al., 1993). At the level of growth plate, the perichondrial ossification groove of Ranvier surrounds the epiphyseal cartilage. It contains different groups of cells, among them chondroblast precursors, which may contribute to the widening of the epiphysis via appositional chondrogenesis (Shapiro et al., 1977).

The morphogenetic events of endochondral bone formation are accompanied by characteristic changes in the matrix composition. The initial mesenchymal matrix of the limb bud mainly contains evenly distributed types I and III collagen, and fibronectin. With the onset of mesenchymal condensation into the cartilaginous template, these proteins concentrate in the core and, for a short time, are co-expressed with the cartilage specific type II collagen (Dessau et al., 1980). With overt chondrogenesis, the expression of mesenchymal markers ceases and chondrocytes switch to synthesize and secrete cartilaginous markers. Matrix com- 
ponents of a typical hyaline cartilage can be divided into three groups: collagens (Bruckner and van der Rest, 1994), proteoglycans (Roughley and Lee, 1994) and noncollagenous glycoproteins (Neame et al., 1999). The bulk of the cartilaginous matrices build up from the aggregates of the cartilage specific, large chondroitin sulfate proteoglycan, aggrecan bound to hyaluronan and link protein which are embedded in a mesh of collagen fibrils. The fibrils consist of mainly type II collagen but also contain types IX and XI collagen as minor components. The physical properties of the cartilage depend on these suprastructural elements of the matrix. Aggrecan has a high charge density due to its anionic glycosaminoglycan (GAG) chains, which leads to water binding and generation of an internal hydrostatic pressure that resists compressive forces. The fibrillar network contains the swelling pressure exerted by the proteoglycan molecules and provides tensile strength and resistance against shear forces. The cartilage matrix contains additional collagen and proteoglycan molecules. Collagen VI forms beaded filaments in articular cartilage, whereas collagen $\mathrm{X}$ is expressed by hypertrophic chondrocytes and might be involved in matrix mineralization. Small proteoglycans such as fibromodulin and decorin are associated with collagen fibrils and are thought to regulate fibril diameter. The functions of noncollagenous, nonproteoglycan macromolecules of cartilage, such as chondroadherin, cartilage oligomeric protein, matrilin-1 and matrilin-3, are less well known but these molecules may play roles in matrix assembly and cellmatrix interactions.

The matrix molecules of the developing bones are organized into well defined ultrastructural compartments (for review see Hunziker, 1994). The narrow pericellular compartment directly surrounding the chondrocytes is rich in aggrecan and contains extremely fine collagen fibrils. The territorial matrix compartment consists of a fine collagen network bordering the individual cells or cell groups in the growth plate. The interterritorial matrix between the cell columns is characterized by thicker, longitudinally oriented collagen bundles. The interfibrillar space, both in the territorial and interterritorial compartments, is filled with proteoglycans and noncollagenous macromolecules.

\section{Lethal phenotypes caused by mutations in genes encoding cartilage extracellular matrix proteins}

\section{A. Achondrogenesis type II-hypochondrogenesis: man and mouse}

The major structural components of the cartilage extracellular matrix are heterotypic fibrils of collagens II, XI and IX. The predominant collagen in these fibrils is collagen II, composed of three identical $\alpha 1$ (II) chains encoded by the COL2A1 gene (Kielty et al., 1993) Collagen XI, $[\alpha(\mathrm{XI}) \alpha 2(\mathrm{XI}) \alpha 3(\mathrm{XI})]$ copolymerises with collagen II, and is buried within the fibril providing a regulatory mechanism for fibril diameter (Eyre et al., 1987). The $\alpha 3(\mathrm{XI})$ chain is also a product of COL2A1, but undergoes different posttranslational modifications. Collagen IX is associated with the surface of the collagen II/XI fibrils and is thought to play a role in modifying the interactions of the fibrils with one another and with other matrix components (Bruckner and van der Rest, 1994).

Collagen II mutations produce a spectrum of chondrodysplasias ranging from mildly affected individuals of normal stature and premature osteoarthritis (Stickler syndrome), to severely affected patients with disproportionately short stature (spondyloepiphyseal dysplasia congenita and Kniest dysplasia) and the lethal forms of achondrogenesis type II and hypochondrogenesis where affected individuals have severe limb and truck shortening and grossly abnormal cartilage structure and endochondral bone formation (Kuivaniemi et al., 1991). These more severe chondrodysplasias result from dominant negative mutations, most commonly glycine substitution mutations in the triple helical obligatory Gly-X-Y motif, which interfere with the integrity of the triple helical domain. Helix formation is delayed, leading to increased post-translational modification of lysine residues within the helical domain and reduced secretion concomitant with increased intracellular degradation.

Recent studies have shown that patients with achondrogenesis and hypochondrogenesis resulting from heterozygous collagen II helix mutations, Gly ${ }^{769}$ to Ser (Chan et al., 1995) and Gly ${ }^{913}$ to Cys (Mundlos et al., 1996), have no detectable collagen II protein in the cartilage, but express collagens I and III instead. Detailed biochemical analysis provided an explanation for the absence of both mutant and normal collagen II in the cartilage (Chan et al., 1995; Mundlos et al., 1996) While the mutant COL2A1 allele was transcribed and translated, the mutant $\alpha 1$ (II) exerted a dominant negative effect on collagen II biosynthesis, resulting in disruption of helix formation, intracellular retention and excessive postranslational modification of mutant-containing collagen II trimers, which were degraded intracellularly (Chan et al., 1995). It is important to note that with equal mutant and normal allele expression, 7/8 of the collagen II trimers will contain at least one mutant chain and would be directly targeted for destruction. The combination of the effects of this stimulation of intracellular degradation pathways and the consumption of a proportion of the normal COL2A1 allele product as $\alpha 3(\mathrm{XI})$ in collagen XI accounts for the absence of collagen II from the cartilage matrix in vivo.

It is of interest to compare the clinical and biochemical phenotypes of these patients with a collagen II null-mice ( $\mathrm{Li}$ et al., 1995; Aszódi et al., 1998a). The homozygous null mice die around birth due to breathing inability associated with cleft palate. Mutant animals are dwarfs with severe deformities of the endoskeleton similar to human achondrogenesis type II (Fig . 2). The long bones are shortened, the 


\section{Achondrogenesis type II}

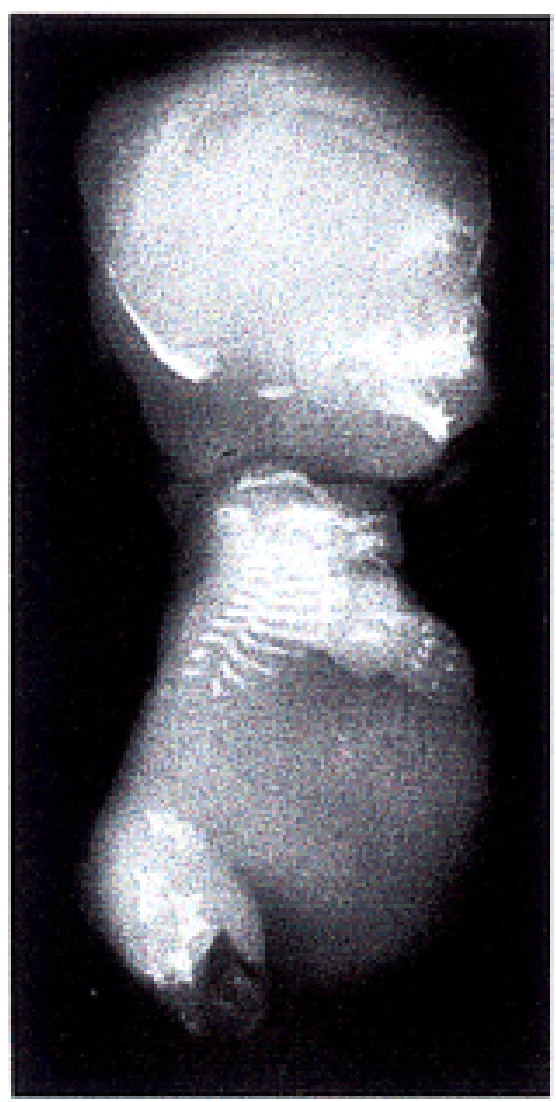

\section{Mouse model}
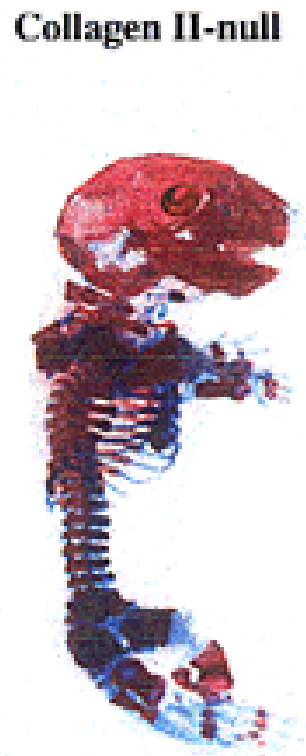

wild type

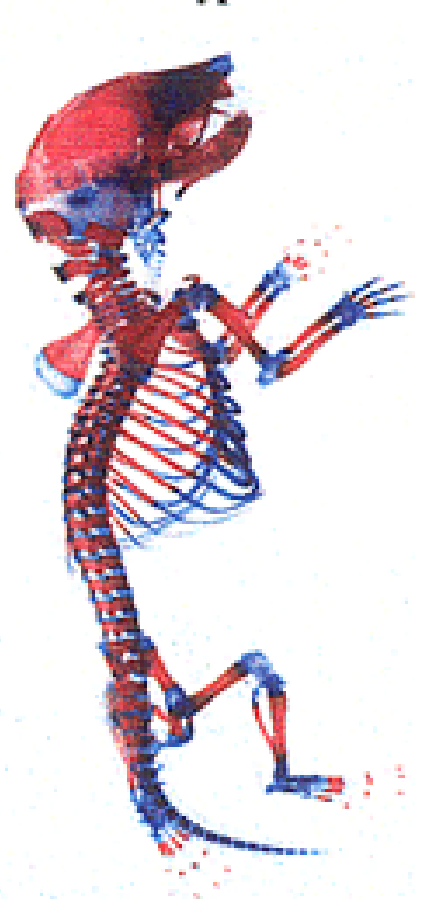

Fig. 2. Lack of type II collagen in cartilage ECM leads to lethality and severe skeletal abnormalities both in man and mouse. Radiograph of a proband with achondrogenesis type II and skeletal staining of a newborn collagen II-null mouse show similar skeletal defects: short limbs, ribs and trunk, and domed skull.

thorax is narrowed, the vertebral bodies and ribs are abnormal in shape. All endochondral bones are soft and hyaline cartilages are structurally disorganized. Histological analysis of long bones reveals unusual anisotropic chondrocytes in the epiphysis and the complete lack of organized growth plate. However, hypertrophic-like cells are detectable adjacent to the chondroosseous junction. The metaphyseal/diaphyseal parts of the long bones are greatly reduced and show insufficient trabecular bone formation. Our immunohistochemical studies demonstrated a significant amount of types I and III collagen throughout the cartilage (Aszódi et al., 1998a). Using in situ hybridization, we observed a subpopulation of mutant chondrocytes expressing both collagens I and III transcripts although, we cannot exclude that the immunodetectable types I and III collagens are partially derived from the perichondrium/periosteum.

Furthermore, studies on the collagen II-null mouse demonstrated that collagen II is essential to allow removal of the notochord and in the formation of the intervertebral discs (IVD) (Aszódi et al., 1998a). IVD is composed of the central, proteoglycan rich nucleus pulposus and the peripherial annulus fibrosus. The nucleus pulposus is derived from the notochord, whereas the annulus fibrosus differentiates from the intervertebral mesenchyme. During normal mammalian development the early notochord is a rod-like structure extending through the entire trunk region. Later on, the notochord gradually vanishes from the areas of vertebral bodies and expands between the vertebrae forming the discoid-like nucleus pulposus. In collagen II-null mice the notochord is not removed from the vertebral segments and persists as a rod until birth. We suggest that the lack of notochordal reorganization is the consequence of the mechanically weak cartilage of vertebral bodies, which is unable to exert proper mechanical forces essential for the initiation of notochordal removal. Interestingly, abnormal IVD development has not been reported in patients with achondrogenesis type II/hypochondrogenesis. This discrepancy may be explained by species specific differences or, more likely, this abnormality has escaped detection in the human condition.

The data from these studies provide direct evidence that in the absence of collagen II, collagen I/III fibrils are clearly unable to produce a functional cartilage matrix. This dem- 
onstrates the importance of collagen II in establishing the correct temporal and spatial organisational relationships with other components of the cartilage extracellular matrix critical for cartilage function and normal endochondral ossification. However, analysis of cartilage from patients and the homozygous Col2a1-null mice demonstrated that despite this lack of collagen II, the chondrocytes expressed other cartilage specific collagen gene products such as $\alpha 1(\mathrm{IX}), \alpha 2$ (IX), $\alpha 1(\mathrm{XI}), \alpha 2(\mathrm{XI})$, and were able to follow their programmed differentiation and maturation pathway to hypertrophy (Chan et al., 1995). In the mouse model the maintenance of the chondrocyte phenotype in the absence of collagen II was further confirmed by the detection of normal expression pattern of a wide range of cartilage-specific matrix protein genes (Aszódi et al., 1998a)

The molecular mechanisms driving the ectopic expression of collagens I and III in cartilage are unknown. One possible mechanism suggested by our studies is that continual collagen II signalling to the cell is required to suppress inappropriate collagen I/III expression by differentiated chondrocytes. In the absence of collagen II, a default collagen I/III biosynthetic program is switched on. The inability of chondrocytes grown in alginate culture matrix to switch off collagen I/III synthesis (Kern et al., 1991) shows that the collagen II effect is specific and not simply related to the inability of the collagen I/III matrix to provide the three-dimensional network previously shown to be critical for chondrocyte phenotypic stability (Benya and Shaffer, 1982). It is possible that this regulatory effect is directed via specific cell surface receptors such as the integrins (Hynes, 1992) which normally bind to collagen II.

The comparison of subtle differences in the biochemical phenotype of the collagen II-null mouse and achondrogenesis/hypochondrogenesis patients suggests some intriguing possiblilities about the role of collagen XI in fibrillar organisation. In the mutant mouse the collagen $\mathrm{XI}[\alpha 1(\mathrm{XI}) \alpha 2(\mathrm{XI})]$ heterotrimer was unstable and sensitive to pepsin digestion, demonstrating that the contribution of the $\alpha 3(\mathrm{XI})$ chain by the COL2A1 gene is critical for stable collagen XI triple helix formation (Aszódi et al., 1998a). The ectopically expressed collagens I and III were readily extracted by $4 \mathrm{M}$ guanidine, a procedure that does not solubilise organised crosslinked collagen fibrils (Chan et al., 1995) and ultrastructural analysis further confirmed the sparsity, abnormal organisation and appearance of the collagen I/III fibrils (Aszódi et al., 1998a). However, a different result was seen for the ectopically expressed collagens I/III expressed in cartilage from achondrogenesis/hypochondrogenesis patients. In these cartilages the collagens were not extracted by $4 \mathrm{M}$ guanidine, but required pepsin digestion suggesting a more normal organised and crosslinked mature collagen I/ III fibril network (Chan et al., 1995; Mundlos et al., 1996).

These studies on collagen II-null mice, and on functionally null chondrodysplasia patients, provide some important new insights in the role collagen in skeletogenesis. They demonstrate that chondrocyte differentiation, maturation and hypertrophy can occur in the absence of collagen II expression, but that the collagen II matrix is critical for cartilage organisation and function. Without the supramolecular structure of the collagen II matrix, endochondral ossification and other developmental processes that involve cartilaginous precursor steps, such as IVD formation, are severely impaired.

\section{B. Critical role of perlecan in skeletal development}

Perlecan is a large secreted proteoglycan that is highly expressed during development as well as in adult tissues (Hassell et al., 1980; Murdoch et al., 1994). Initially, it was believed that the expression of this proteoglycan is restricted to basement membranes, but more recent studies showed a high perlecan expression in developing cartilage (Handler et al., 1997; French et al., 1999).

Perlecan is known to interact with a number of ECM components including collagen IV, laminin, nidogens and fibronectin, various growth factors and cellular receptors such as integrins and $\alpha$-dystroglycan. To date, nothing is known about the binding partners of perlecan in the cartilage ECM. In contrast to most of the other proteoglycans in cartilage which contain chondroitin and/or keratin sulfate GAG chains, perlecan is mainly substituted by heparan sulfate (HS) side chains. In other tissues, the HS side chains bind FGF-2 and promote its receptor binding and biological activity (Aviezer et al., 1994), although whether perlecan plays this role in cartilage is not known.

Although perlecan is a prominent component of the cartilage ECM, its in vivo importance for cartilage development was unclear until mice carrying a null mutation in the perlecan gene were generated. Perlecan-null mice display multiple abnormalities including a severe defect in skeletal development (Costell et al., 1999; Arikawa-Hirasawa et al., 1999). Many of the perlecan-null embryos die at mid-gestation due to heart failure (Costell et al., 1999). The surviving embryos develop a severe chondrodysplasia between E15 and the newborn stage, characterized by disproportionate dwarfism with short limbs, neck and snout. In addition, the spine is short and displays severe kyphoscoliosis, the thorax is narrow, and the ribs, vertebrae, and long bones are malformed. Perlecan-null mice have a cleft palate and die at birth due to respiratory failure. Some mutants lack calvarial bones and show exencephaly due to abnormal expansion of the neuroepithelium (Costell et al., 1999).

The lack of perlecan disrupts endochondral ossification, resulting in abnormal development of all endochondral bones. The initial formation of the cartilage anlage is normal and the first visible abnormalities start at E13-14 when the perlecan-null long bones were shorter, thicker and bent compared to wild type. The underlying cause is a severe disorganization in the perlecan-null epiphyseal growth plates that lack the typical columnar arrangement of the prolifera- 
tive and the hypertrophic chondrocytes. Earlier in vitro studies suggested that perlecan both promotes and maintains chondrocyte differentiation (French et al., 1999). In contrast, our data clearly show that perlecan-null chondrocytes express lineage-specific markers including Sox9, indian hedgehog and type $\mathrm{X}$ collagen, indicating the differentiation cascade is not disturbed and that true hypertrophic chondrocytes are formed in the perlecan-null cartilage (Costell et al., 1999).

Instead, we have evidence that the cartilage matrix is affected in the absence of perlecan cartilage (Costell et al., 1999). Immunohistochemical studies of perlecan-null cartilage show that all the examined matrix components are present such as collagen types II, IX, X, aggrecan, matrilin1 and -3 , and COMP. Safranin-O staining of the mutant cartilage, however, was less than controls, indicating reduced glycosaminoglycan levels. Moreover, ultrastructural analyses revealed that growth plate cartilage in mutants lacks the characteristic collagen network, and that the collagen fibrils are fewer and shorter in length. The matrix surrounding the mutant hypertrophic chondrocytes showed either minimal or no mineral deposits. In addition, the calcified trabeculae were transversely orientated in perlecan-null growth plates in contrast to longitudinal orientation in controls, suggesting that a highly organized cartilage matrix is a prerequisite for proper endochondral bone formation. Ultrastructural examination also revealed that hypertrophic chondrocytes had an abnormal morphology with increased density of organelles, distended ER and enrichment of cytosolic ribosomes and polysomes, indicating that the perlecan-null hypertophic chondrocytes have a high biosynthetic activity. This is further supported by the fact that these chondrocytes have increased message levels for cartilage ECM genes such as Col2a1, matrilin-3 and COMP.

Taken together, these data suggest that perlecan is essential for maintaining the macromolecular network of ECM proteins in cartilage. An underlying mechanism may be that perlecan protects the cartilage ECM from degradation by proteases, either by masking the components or by binding and thereby inactivating cartilage proteases. A similar action of perlecan has previously been described. Perlecan was shown to inhibit proteolytic degradation of $\beta$-amyloid fibrils thereby contributing to accumulation and persistence of neurotoxic plaques in Alzheimers disease (Gupta-Bansal et al., 1995). Another possibility is that perlecan modulates signaling pathways regulating chondrocyte proliferation and hypertrophy. A possible candidate would be FGFR3, a negative regulator of chondrocyte proliferation and hypertrophy, shown to be mutated in some cases of human skeletal dysplasias. Perlecan may, due to its growth factor binding abilities, regulate FGFR3 activity by sequestering its ligands. Aberrant FGFR3 signaling due to increased FGFR3 and FGF1 expression has been suggested by ArikawaHirasawa et al. (1999), although we did not detect increased FGFR3 expression in the cartilage of our mutant mice (Cos- tell et al., 1999; unpublished observation).

\section{Mild or lack of phenotype after ablation of cartilage matrix protein genes}

\section{A. Collagen fibril-associated proteins (CAPs)}

Collagen fibrillogenesis, as well as the interaction of the collagen fibrils with each other or with the surrounding matrix constituents, might be regulated by macromolecules associated with the fibrillar surface. These molecules include type IX collagen, small leucine-reach proteoglycans such as decorin, fibromodulin and lumican, and matrilin-1. Although numerous in vitro experiments suggest the importance of CAPs in matrix assembly, their in vivo roles have not been completely clarified.

Besides type XI collagen, collagen IX is the other minor component of the cartilage collagen fibrils (for review see Bruckner and van der Rest, 1994). Collagen IX molecules constitute about $10 \%$ of the total collagen content of fetal cartilage and $1-2 \%$ of that in adult articular cartilage. Type IX collagen is a heterotrimeric molecule composed of three polypeptide chains, [ $\alpha 1$ (IX), $\alpha 2($ IX), $\alpha 3$ (IX)]. Each chain is encoded by a distinct gene (Co19a1, Co19a2, Co19a3) and contains three triple helical domains (COL1-COL3) interrupted by four non-triple helical domains (NC1-NC4). In hyaline cartilage, the $\mathrm{NC}$ domains are short stretches of less than 30 residues, except the large $\mathrm{N}$-terminal globular domain (NC4) of the $\alpha 1$ (IX) chain which contains 243 residues. In other tissues where collagen IX is expressed, such as vitreous humor, avian primary corneal stroma and the notochord, the $\alpha 1$ chain carries only a 3 -amino acid NC4 domain due to the use of an alternative, downstream promoter of the Col9al gene (Nishimura et al., 1989). It has been shown that cartilage type IX collagen molecules are covalently cross-linked to type II collagen, as well as to each other, suggesting a pivotal contribution of type IX collagen to the assembly and stability of the collagen network (Wu et al., 1992; Diab et al., 1996). Type IX collagen can also be considered as a proteoglycan since it can carry a chondroitin sulfate chain covalently attached to the NC3 domain of the $\alpha 2$ (IX) chain (McCormick et al., 1987). In addition, the NC4 domain of the $\alpha 1$ (IX) chain may interact with glycosaminoglycans in cartilage suggesting that type IX collagen molecules might mediate the interaction of collagen fibrils with other components of the extracellular matrix.

Generation of transgenic mice lacking both the long and short isoforms of the $\alpha 1$ (IX) chain partially supports the importance of type IX collagen in cartilage function (Fässler et al., 1994). Whereas mice homozygous for the Col9al gene deletion grow without apparent abnormalities in their skeleton until early adulthood, older animals develop an degenerative disease of knee joints. The first sign of pathological changes were observed in 4-month-old mice. Although tibial and femoral articular cartilages were morphologically 
A

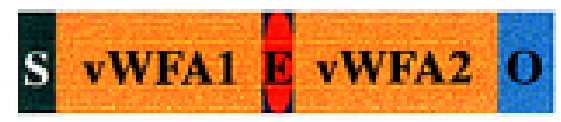

B

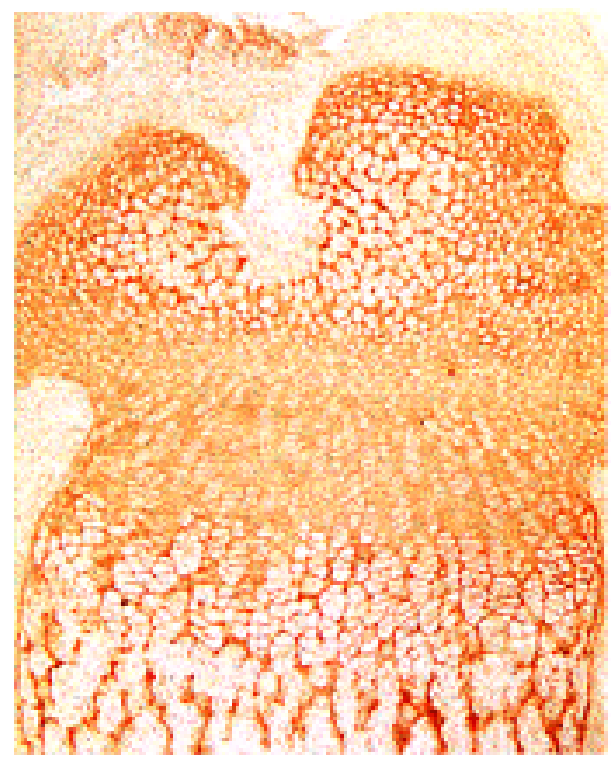

matrilin-3
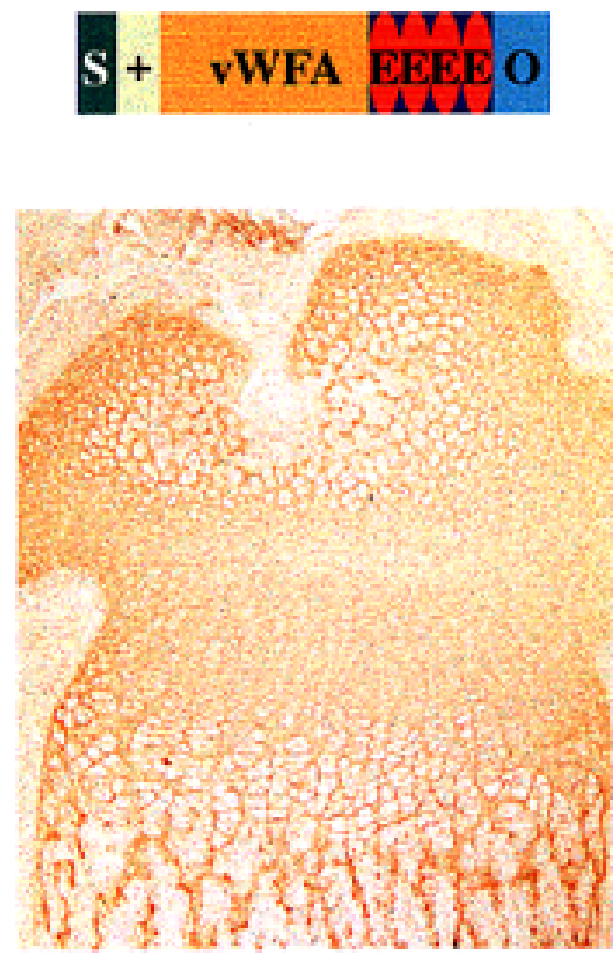

Fig. 3. The similar structure and expression pattern of mouse matrilin-1 and matrilin-3 suggests redundancy during skeletal development. (A) Domain structures of matrilin-1 and matrilin-3. Matrilin-1 precursor contains a signal peptide (S), two von Willebrand factor A-like domains (vWFA1 and vWFA2) separated by one epidermal growth factor-like module (E) and a C-terminal, coiled-coil oligomerization motif (O). Matrilin-3 is composed of only one vWFA domain, four EGF-like modules, one oligomerization motif and, in addition, a positively charged domain (+) following the signal peptide. (Modified after Deák et al., 1999). (B) Immunohistochemical stainings for matrilin-1 and matrilin-3 in the humerus of a newborn mouse. Note the identical deposition of matrilin-1 and matrilin-3 in cartilage.

normal, we found a decrease in cartilage proteoglycan content in mutant mice compared to wild type animals demonstrated by Safranin Orange staining. Proteoglycan depletion is a characteristic feature of the early onset osteoarthritis which is usually followed by progressive degeneration of the articular cartilage. Indeed, mutant mice older than 9 months showed extensive alteration of the joints characterized by fibrillation of the surface, appearance of pyknotic nuclei and finally, the complete loss of articular cartilage in some areas. These abnormalities resulted in prominent shape changes in the joints. The tibial cartilage surfaces were concave and the femuropatellar grooves were $\mathrm{V}$ shaped, while in wild type animals they were convex and Ushaped, respectively.

The normal sequence of endochondral bone formation in mutant embryos and young animals was somewhat surprising. A possible explanation could be that the $\alpha 2$ (IX) and $\alpha 3$ (IX) chains may form homo- and/or heterotrimers which can associate with the collagen fibrils and compensate for the lack of $\alpha 1$ (IX) chain during development. However, we recently showed that although Col9a2 and Col9a3 are transcribed in Col9a1-/- mice, the corresponding $\alpha 2$ (IX) and $\alpha 3$ (IX) chains are not present in cartilage extracts of mutant animals (Hagg, et al., 1997). Furthermore, the type II/XI collagen fibrils are indistinguishable in shape and banding pattern from those of control mice. Taken together, these data demonstrate that type IX collagen is not essential for skeletal morphogenesis and proper fibril formation but it is important for the functional and mechanical stability of the weight-bearing articular cartilages. This conclusion is further supported by the similar phenotype of transgenic mice expressing truncated $\alpha 1$ (IX) chains (Nakata et al., 1993). In addition, human patients with mutations either in COL9A2 or COL9A3 genes develop early-onset osteoarthritis which predominantly affects the knee joints (Muragaki et al., 1996; Holden et al., 1999; Paassilta et al., 1999). More recently, a mutation in the COL9A1 gene, that converts a codon for glutamine to tryptophan, has been found in patients with intervertebral disc disease characterized by sciatica and herniation (Annunen et al., 1999). We found no evi- 
dence for abnormal vertebral column development in collagen IX-deficient animals (Fässler et al., 1994). However, mild degenerative changes of the spine were reported in transgenic mice with the dominant-negative Col9al mutation (Kimura et al., 1996).

Matrilin-1, also named cartilage matrix protein (CMP), is an other example of a molecule which may mediate interactions between collagen fibrils and other constituents of the cartilage extracellular matrix. It belongs to the matrilin family of ECM proteins, which so far consists of four members (matrilin-1, -2, -3, and -4). Matrilins are multidomain proteins consisting of von Willebrand factor A (vWFA)-like domain(s), epidermal growth factor (EGF)-like domain(s) and a coiled-coil $\alpha$-helical motif (for review see Deák et al., 1999). Matrilin-1 and -3 are predominantly expressed in hyaline cartilage, whereas matrilin- 2 and -4 have a wider tissue distribution. Matrilin-1 is a homotrimer of disulfidebonded subunits which assemble via their C-terminal coiled coil domains. In addition to the coiled coil domain, each monomer consist of two vWFA modules separated by one EGF-like motif. Interestingly, heterooligomers of matrilin-1 and matrilin-3 have been recently found in bovine but not in mouse epiphyseal cartilage (Wu and Eyre, 1998; Aszódi et al., 1999). Matrilin-1 is an abundant component of tracheal, nasal septal, epiphyseal and auricular cartilage but absent in normal mammalian articular cartilage. In mouse, the protein is detectable throughout the growth plate, whereas matrilin1 transcript is expressed in the resting, proliferative and upper hypertrophic zones but not in the lower hypertrophic region (Aszódi et al., 1996). Matrilin-1 can covalently attach to aggrecan core protein and the proportion of such bound molecules increases with cartilage maturation and aging (Hauser et al., 1996). Because matrilin-1 is also associated with collagen fibrils (Winterbottom et al., 1992) it may have an integrative function in cartilage connecting the two major supramolecular structures to each other.

Surprisingly, mice harboring the inactivated $\mathrm{Crtm}$ gene encoding matrilin-1 have no apparent phenotype (Aszódi et al., 1999). Homozygous mutant mice are viable, fertile and show no skeletal abnormalities either on a histological or ultrastructural level. Furthermore, biochemical analysis demonstrated normal collagen maturation and cross-linking in the mutant cartilage. These data show that matrilin-1 plays no essential role in endochondral bone formation, collagen fibrillogenesis and proper cartilage function in vivo. Taking into account the similar structure and cartilage specific expression pattern of matrilin-1 and matrilin-3 (Fig. 3, Wagener et al., 1997, Aszódi et al., 1999) we suggest that the function of these matrilins might be redundant during skeletogenesis explaining the lack of phenotype in matrilin1 deficient mice. The fact that neither human nor naturally occuring mouse skeletal disorders have been attributed to mutations in the genes coding for matrilin-1 or matrilin-3 further supports this hypothesis (Aszódi et al., 1998b;
Wagener et al., 2000).

Similar to matrilin-1, the lack of skeletal phenotype of the fibromodulin-null mice (Svensson et al., 1999) might be due to redundant mechanisms. Fibromodulin belongs to the family of proteoglycans sharing the leucine-rich repeat (LRR) motif, which so far consists of 9 members (for review see Iozzo, 1999). Leucine-rich proteoglycans (LRPs) are expressed in many tissues including skin, tendon, cornea, cartilage and bone. Some of them, like fibromodulin, decorin and lumican, bind to collagen fibers and retard collagen I or collagen II fibrillogenesis in vitro (Vogel and Trotter, 1987). Recent gene targeting experiments indicate that these molecules have fundamental roles in regulating collagen fibril formation in vivo, at least in certain tissues. The fibromodulin-deficient mice show altered tendon structure due to the high proportion of thin, irregular collagen fibrils (Svenssson et al., 1999), whereas decorin-null mice have fragile skin with abnormally thick collagen fibrils (Danielson et al., 1997). Lumican knockout mice also develop skin fragility, and in addition, corneal opacity due to abnormal collagen fibril formation. (Chakravarty et al., 1998). Although, fibromodulin, decorin and lumican are present in fetal and articular cartilages, the corresponding mutant mice show no skeletal abnormalities. It is possible that these LRPs might not play a significant role in type II collagen fibrillogenesis, or the lack one of the LRP members in cartilage is efficiently compensated for by other member(s).

\section{B. Normal skeletal development without Tenascin-C}

Tenascin- $\mathrm{C}$ is a large, hexameric extracellular matrix protein which is highly expressed during embryogenesis at special locations such as the sites of epithelial-mesenchymal interactions and along migratory pathways of neural crest cells. It posseses both anti-adhesive and adhesive properties, binds to different cell surface receptors and is thought to be important for various developmental processes including chondrogenesis (for review see Pacifici, 1994; Mackie and Murphy, 1998). Tenascin-C is present in the condensing mesenchyme and the newly formed cartilage anlage but later on, it is largely eliminated from the developing endochondral bones except the perichondrium and the periphery of epiphyses (Mackie et al., 1987). Tenascin-C is also found in condensing mesenchyme of intramembranous bones, on the periosteal and endosteal surfaces but not in the mature bone matrix (Mackie et al., 1987). In adult, tenascin-C is abundant in permanent cartilages such as articular cartilage and tracheal rings (Pacifici et al., 1993). It has been shown in vitro that tenascin-C promotes cartilage nodule formation from chick limb-bud mesenchymal cells, whereas anti-tenascin-C antibodies inhibit it (Mackie et al., 1987, Choung et al., 1993). The expression pattern of tenascin-C as well as the in vitro data suggest that tenascin- $\mathrm{C}$ is involved in chondrogenic and osteogenic differentiation, and important for 
articular cartilage development (Pacifici, 1994). However, tenascin-C-deficient mice generated independently in our laboratory (Forsberg et al., 1996) and by others (Saga et al., 1992) displayed no apparent skeletal, or other abnormalities indicating that tenascin- $\mathrm{C}$ is not essential for development and normal tissue function. Furthermore, we demonstrated that tenascin-C has no critical role during tissue repair processes such as cutaneous wound healing and peripherial nerve regeneration (Forsberg et al., 1996), and tumor growth and metastasis (Talts et al., 1999). Although recent studies have shown that, depending on the genetic background or under specialised pathological interventions, tenascin-C knockout mice display some abnormalities (reviewed by Mackie and Tucker, 1999), skeletal defects have not been reported.

\section{Conclusions}

Extracellular matrix molecules define the structure and physical properties of connective tissues. Mouse strains with spontaneous and experimentally induced mutations in genes coding for ECM proteins provide an unique opportunity to study the roles of these molecules during development, in normal tissue function, and help to clarify their involvement in human heritable disorders (reviewed by Aszódi et al., 1998c). Our knockout mouse lines bearing null mutations in genes encoding cartilage ECM constituents suggest that some matrix molecules have an essential role during the formation of the skeleton, whereas others are apparently less important. The availability of transgenic models might help to develop effective gene therapeutic tools. We must note, however, that our animal models are not completely identical with the corresponding human skeletal disorders. This may be due to species specific differences or alternatively, may be due to the fact that many of the human conditions result from dominant negative effects rather than true null mutations as we have produced in the mouse.

The more detailed analyses that can be conducted on mice in comparison with patients may well reveal novel aspects of pathology in the human conditions. For example, it has not been reported that humans with achondrogenesis type II fail to remove the notocord during spine development but this is clearly the case in the collagen II-null mouse. In addition, the demonstration of the importance of perlecan for mouse skeletal development makes this gene a new potential candidate for heritable human skeletal pathologies of, as yet, unknown origin.

\section{References}

Annunen S., Paassilta, P., Lohiniva, J., Perälä, M., Plihkajamaa, T., Karppinen, J., Tervonen, O., Kröger, H., Lähde, S., Vanharanta, H., Ryhänen, L., Göring, H.H.H., Ott, J., Prockop, D.J., and Ala-Kokko, L. 1999. An allele of COL9A associated with intervertebral disc disease.
Science, 285: 409-412.

Arikawa-Hirasaw, A.E., Watanabe, H., Takami, H., Hassell, J.R., and Yamada, Y. 1999. Perlecan is essential for cartilage and cephalic development. Nat. Genet., 23: 354-358.

Aszódi A., Hauser, N., Studer, D., Paulsson, M., Hiripi, L., and Bösze, Z. 1996. Cloning, sequencing and expression analysis of mouse cartilage matrix protein cDNA. Eur. J. Biochem., 236: 970-977.

Aszódi, A., Chan, D., Hunziker, E., Bateman, J.F., and Fässler, R. 1998a. Collagen II is essential for the removal of the notochord and the formation of intervertebral discs. J. Cell Biol., 143: 1399-1412.

Aszódi, A., Beier, D., Hiripi, D., Bösze, Z., and Fässler, R. 1998b. Sequence, structure and chromosomal localization of Crtm gene encoding mouse cartilage matrix protein and its exclusion as a candidate for murine achondroplasia. Matrix Biol., 16: 563-573.

Aszódi, A., Pfeifer, A., Wendel, M., Hiripi, L., and Fässler, R. 1998c. Mouse models for extracellular matrix diseases. J. Mol. Med., 76: 238252.

Aszódi, A., Bateman, J.F., Hirsch, E., Baranyi, M., Hunziker, E.B., Hauser, N., Bösze, Z., and Fässler, R. 1999. Normal skeletal development of mice lacking matrilin 1: redundant function of matrilins in cartilage? Mol. Cell Biol., 19: 7841-7845.

Aviezer, A., Hecht, D., Safran, M., Eisnger, M., David, G. and Yaynon, A. 1994. Perlecan, basal lamina proteoglycan, promotes basoc fibroblast growth factor-receptor binding, mitogenesis, and angiogenesis. Cell, 79: 1005-1013.

Benya, P.D. and Shaffer, J.D. 1982. Dedifferentiated chondrocytes reexpress the differentiated collagen phenotype when cultured in agarose gels. Cell, 30: 215-224.

Bianco, P., Cancedda, F.D., Riminucci, M. and Cancedda, R. 1998. Bone formation via cartilage models: the "borderline" chondrocyte. Matrix Biol., 17: 185-192.

Bruckner, P. and van der Rest, M. 1994. Structure and function of cartilage collagens. Microsc. Res. Technique, 28: 378-384.

Chakravarty, S., Magnuson, T., Lass, J.H, Jepsen, K.J., LaMantia, C., and Carrol, H. 1998. Lumican regulates collagen fibril assembly: skin fragility and corneal opacity in the absence of lumican. J. Cell Biol., 141: 1277-1286.

Chan, D., Cole, W.G,. Chow, C.W, Mundlos, S., and Bateman, J.F. 1995. A COL2A1 mutation in achondrogenesis type II results in the replacement of type II collagen by type I and III collagens in cartilage. J. Biol. Chem., 270: $1747-1753$.

Choung, C-M., Widelitz, R.B., Jiang, T.X., Abbot, U.K., Lee, Y.S., and Chen, H.M. 1993. Roles of adhesion molecules NCAM and tenascin-C in limb skeletogenesis: Analysis with antibody perturbation, exogenous gene expression, Talpid ${ }^{2}$ mutants and activin stimulation. In Limb Development and Regeneration (J.F. Fallon, P.F. Goetinck, R.O. Kelley, and D.L. Stocum, eds.). Wiley-Liss, New York, pp. 465-474.

Costell, M., Gustafsson, E., Aszódi A., Mörgelin, M., Bloch, W., Hunziker, E., Addicks, K., Timpl, R., and Fässler, R. 1999. Perlecan maintains the integrity of cartilage and some basement membranes. J. Cell Biol., 147: $1109-1122$.

Danielson, K.G., Baribault, H., Holmes, D.F., Graham, H., Kadler, K.E., and Iozzo, R.V. 1997. Targeted disruption of decorin leads to abnormal collagen fibril morphology and skin fragility. J. Cell Biol., 136: 729743.

Deák, F., Wagener, R., Kiss, I., and PauLsson, M. 1999. The matrilins: a novel family of oligomeric extracellular matrix proteins. Matrix Biol., 18: 55-64.

Dessau, W., von der Mark, H., von der Mark, K., and Fischer, S. 1980. Changes in the patterns of collagens and fibronectin during limb-bud chondrogenesis. J. Embryol. Exp. Morph., 57: 51-60.

Diab, M., Wu, J.J., and Eyre, D.R. 1996. Collagen type IX from human cartilage: a structural profile of intermolecular cross-linking sites. Bio- 
chem. J., 314: 327-332.

Erlebacher, A., Filvaroff, E.H., Gitelman, S.E., and Derynck, R. 1995. Toward a molecular understanding of skeletal development. Cell, 80: $371-$ 378.

Eyre, D.R., Apon, S., Wu, J.J., Ericsson, L.H., and Walsh, K.A. 1987. Collagen type IX: evidence for covalent linkages to type II collagen in cartilage. FEBS Lett., 220: 337-341.

Fässler, R., Schnegelsberg, P.N.J., Dausman, J., Shinya, T., Muragaki, Y., McCarthy, M.T., Olsen, B.R., and Jaenisch, R. 1994. Mice lacking $\alpha 1$ (IX) collagen develop noninflammatory degenerative joint disease. Proc. Natl. Acad. Sci. USA, 91: 5070-5074.

Forsberg, E., Hirsch E., Fröhlich, L., Meyer, M., Ekblom, P., Aazódi, A., Werner, S., and Fässler, R. 1996. Skin wounds and severed nerves heal normally in mice lacking tenascin-C. Proc. Natl. Acad. Sci. USA, 93: 6594-6599.

French, M.M., Smith, S.E., Akanbi, K., Sanford, T., Hecht, J., FarachCarson, M.C., and Carson, D.D. 1999. Expression of the heparan sulfate proteoglycan, perlecan, during mouse embryogenesis and perlecan chondrogenic activity in vitro. J. Cell Biol., 145: 1103-1115.

Gupta-Bansal, R., Frederickson, R.C., and Brunden, K.R. 1995. Proteoglycan-mediated inhibition of $\mathrm{A} \beta$ proteolysis. A potential cause of senile plaque accumulation. J Biol. Chem., 270: 18666-18671.

Hagg, R., Hedbom, E., Möllers, U., Aszódi, A., Fässler, R., and Bruckner, P. 1997. Absence of the $\alpha 1$ (IX) chain leads to a functional knock-out of the entire collagen IX protein in mice. J. Biol. Chem., 272: 2065020654.

Handler, M., Yurchenco, P.D., and Iozzo, R.V. 1997. Developmental expression of perlecan during murine embryogenesis. Dev. Dyn., 210: 130145.

Hassell, J.R., Gehron-Robey, P., Barrach, H.-J., Wilczek, J., Rennard, S.I., and Martin, G.R. 1980. Isolation of a heparan sulfate-containing proteoglycan from basement membrane. Proc. Natl. Acad. Sci. USA, 77: 4494-4498.

Hauser, N., Paulsson M., Heinegård, D., and Mörgelin, M. 1996. Interaction of cartilage matrix protein (CMP) with aggrecan. Increased covalent crosslinking with maturation. J. Biol. Chem., 271: 32247-32252.

Holden, P., Canty E.G., Mortier, G.R., Zabel, B., Spranger, J., Carr, A., Grant, M.E., Loughlin, J.A., and Briggs, M.D. 1999. Identification of novel pro-alpha2(IX) collagen gene mutations in two families with distinctive oligo-epiphyseal forms of multiple epiphyseal dysplasia. Am. J. Hum. Genet., 65: 31-38.

Horton, W.A. 1993. Morphology of connective tissue: cartilage. In Connective Tissue and its Heritable Disorders. Molecular, Genetic, and Medical Aspects (P.M. Royce, and B. Steinmann, eds.). Wiley-Liss, Inc., New York, pp. 73-84.

Hunziker, E.B., Herrmann, W., Schenk, R.K., Mueller, M., and Moor, H. 1984. Cartilage ultrastructure after high pressure freezing, freeze substitution, and low temperature embedding. I. Chondrocyte ultrastructure. Implications for the theories of mineralization and vascular invasion. $J$. Cell Biol., 98: 267-276.

Hunziker, E.B., Schenk, R.K., and Cruz-Orive, L-M. 1987. Quantitation of chondrocyte performance in growth plate cartilage during longitudinal bone growth. J. Bone Joint Surg., 69: 162-173.

Hunziker, E.B. 1994. Mechanism of longitudinal bone and its regulation by growth plate chondrocyte. Microsc. Res. Technol., 28: 505-519.

Hynes, R.O. 1992. Integrins: versatility, modulation, and signaling in cell adhesion. Cell, 69: 11-25.

Iozzo, R.V. 1999. The biology of the small leucine-rich proteoglycans. $J$. Biol. Chem., 274: 18843-18846.

Kern P., Menasche, M., and Robert, L. 1991. Relative rates of biosynthesis of collagen type I, type V and type VI in calf cornea. Biochem.J., 274 615-617.

Kielty, C.M., Hopkinson, I., and Grant M.E. 1993. Collagen. The collagen family: structure, assembly, and organization in the extracellular matrix. In Connective Tissue and its Heritable Disorders. Molecular, Genetic, and Medical Aspects (P.M. Royce, and B. Steinmann, eds.). Wiley-Liss, Inc., New York, pp. 103-147.

Kimura, T., Nakata, K., Tsumaki, N., Miyamoto, S., Matsui, Y., Ebara, S., and Ochi, T. 1996. Progressive degeneration of articular cartilage and intervertebral discs. An experimental study in transgenic mice bearing a type IX collagen mutation. Int. Orthop., 20: 177-181.

Kuivaniemi, H., Tromp, G., and Prockop, D.J. 1991. Mutations in collagen genes: causes of rare and some common diseases in humans. FASEB J., 5: 2052-2060.

Li, S.W., Prockop, D.J., Helminen, H., Fässler, R., Lapveteläinen, T., Kiraly, K., Peltarri, A., Arakoski, J., Lui, H., Arita, M., and Khillan, J.S. 1995. Transgenic mice with targeted inactivation of the Col $2 \alpha 1$ gene for collagen II develop a skeleton with membranous and periosteal bone but no endochondral bone. Genes. Dev., 9: 2821-2830.

Mackie, E.J. Thesleff, I., and Chiquet-Ehrismann, R. 1987. Tenascin-C is associated with chondrogenic and osteogenic differentiation in vivo and promotes chondrogenesis in vitro. J. Cell Biol., 105: 2569-2579.

Mackie, E.J. and Murphy, L.I. 1998. The role of tenascin-C and related glycoproteins in early chondrogenesis. Microsc. Res. Tech., 15: 102110

Mackie, E.J. and Tucker, R.P. 1999. The tenascin-C knockout revisited. J. Cell Sci., 112: 3847-3853.

McCormick, D., van der Rest, M., Goodship, J., Lozano, G., Ninomiya, Y., and Olsen, B.R. 1987. Structure of the glycosaminoglycan domain in the type IX collagen-proteoglycan. Proc. Natl. Acad. Sci. USA, 84: 4044-4048.

Mundlos, S., Chan, D., Mchgill, J., and Bateman, J.F. 1996. An $\alpha 1$ (II) Gly $^{913}$ to Cys substitution prevents the matrix incorporation of type II collagen which is replaced with type I and III collagens in cartilage from a patient with hypochondrogenesis. Am. J. Med. Genet., 63: 129-136.

Mundlos, S. and Olsen, B.R. 1997a. Heritable diseases of the skeleton. Part I: molecular insight into skeletal development-transcription factors and signaling pathways. FASEB J., 11: 125-132.

Mundlos, S. and Olsen, B.R. 1997b. Heritable diseases of the skeleton. Part II: molecular insight into skeletal development-matrix components and their homeostasis. FASEB J., 11: 227-233.

Muragaki, Y., Mariman, E.C.M., van Beersum, S.E.C., Peräla, M., van Mourik, J.B.A., Warman, M.L., Olsen, B.R., and Hamel, B.J.C. 1996. A mutation in the gene encoding the $\alpha 2$ chain of the fibril-associated collagen IX, COL9A2, causes multiple epiphyseal dysplasia (EDM2). Nat. Genet., 12: 103-105.

Murdoh, A.D., Liu, B., Schwarting, R., Tuan, R.S., and Iozzo, R.V. 1994. Widespread expression of perlecan proteoglycan in basement membranes and extracellular matrices of human tissues as detected by a novel monoclonal antibody against domain III and by in situ hybridization. $J$. Histochem. Cytochem., 42: 239-249.

Nakata, K., Ono, K., Miyazaki, J.I., Olsen, B.R., Muragaki, Y., Adachi, E., Yamamura, K.I., and Kimura, T. 1993. Osteoarthritis associated with mild chondrodysplasia in transgenic mice expressing $\alpha 1$ (IX) collagen chains with a central deletion. Proc. Natl. Acad. Sci. USA, 90: 28702874.

Neame, P.J., Tapp, H., and Azizan, A. 1999. Noncollagenous, nonproteoglycan macromolecules of cartilage. Cell Mol. Life Sci., 55: 13271340 .

Nishimura, I., Muragaki, Y., and Olsen, B.R. 1989. Tissue specific forms of type IX collagen-proteoglycan arise from the use of two widely separated promoters. J. Biol. Chem., 264: 20033-20041.

Paassilta, P., Lohiniva, J., Annunen, S., Bonaventure, J., Le Merrer, M., Pai, L., and Ala-Kokko, L. 1999. COL9A3: a third locus for multiple epiphyseal dysplasia. Am. J. Hum. Genet., 64: 1036-1044.

Pacifici, M., Iwamoto, M., Golden, E.B., Leatherman, J.L, Lee, Y.-S. and 
Chuong, C.-M. 1993. Tenascin is associated with articular cartilage development. Dev. Dyn., 198: 123-134.

Pacifici, M. 1994. Tenascin-C and the development of articular cartilage. Matrix Biol., 14: 689-698.

Roughley, P.J. and Lee, E.R. 1994. Cartilage proteoglycans: structure and potential functions. Microsc. Res. Technol., 28: 385-397.

Saga, Y., Yagi, T., Ikawa, Y., Sakakura, T., and Aizawa, S. 1992. Mice develop normally without tenascin. Genes. Dev., 6: 1821-1831.

Schenk, R.K., Felix, R., and Hofstetter, W. 1993. Morphology of connective tissue: bone. In Connective Tissue and its Heritable Disorders. Molecular, Genetic, and Medical Aspects (P.M. Royce, and B. Steinmann, eds.). Wiley-Liss, Inc., New York, pp. 85-101.

Shapiro, F., Holtrop, M.E., and Glimcher, M.J. 1977. Organization and cellular biology of the perichondrial ossification groove of Ranvier. $J$. Bone Joint Surg., 59-A: 703-723.

Svensson, L., Aszódi, A., Reinholt, F.P., Fässler, R., Heinegård, D., and Oldberg, A. 1999. Fibromodulin-null mice have abnormal collagen fibrils, tissue organization and altered lumican deposition in tendon. $J$. Biol. Chem., 274: 9636-9647.

Talts, J.F., Wirl, G., Dictor, M., Müller, W.J., and Fässler, R. 1999. Tenascin-C modulates tumor stroma and monocyte/macrophage recruitment but not tumor growth or metastasis in a mouse strain with spontaneous mammary cancer. J. Cell. Sci., 112: 1855-1864.

Vanky, P., Brockstedt, U., Hjerpe, A., and Wikstrom, B. 1998. Kinetic studies on epiphyseal growth cartilage in the normal mouse. Bone, 22:
331-339.

Vogel, K.G. and Trotter, J.A. 1987. The effect of proteoglycans on the morphology of collagen fibrils formed in vitro. Coll. Relat. Res., 7: 105-114.

Wagener, R., Kobbe, B., and Paulsson, M. 1997. Primary structure of matrilin-3 gene, a new member of a family of extracellular matrix proteins related to cartilage matrix protein (matrilin-1) and von Willebrand factor. FEBS Lett., 413: 129-134.

Wagener, R., Kobbe, B., Aszódi, A., Liu, Z., Beier, D.R., and Paulsson, M. 2000. Structure and mapping of the mouse matrilin-3 gene (Matn3), a member of a gene family containing a U12-type AT-AC intron. Mamm. Genome, 11: 85-90

Winterbottom, N., Tondravi, M.M., Harrington, T.L., Klier, F.G., Vertel, B.M., and Goetinck, P.F. 1992. Cartilage matrix protein is a component of the collagen fibril of cartilage. Dev. Dyn., 193: 266-276.

Wu, J.J., Woods, P.E., and Eyre, D.R. 1992. Identification of cross-linking sites in bovine cartilage type IX collagen reveals an antiparallel type IItype IX molecular relationship and type IX to type IX bonding. J. Biol. Chem., 267: 23007-23014.

Wu, J.J. and Eyre, D.R. 1998. Matrilin-3 forms disulfide-linked oligomers with matrilin-1 in bovine epyphyseal cartilage. J. Biol. Chem., 273: 17433-17438.

(Received for publication, December 24, 1999

and accepted, December 28, 1999) 